\title{
Prenatal Diagnosis of Placenta Percreta with Ultrasound
}

\author{
Burcu Artunc Ulkumen ${ }^{*}$, Halil Gursoy Pala ${ }^{2}$, Yesim Baytur ${ }^{3}$
}

\begin{abstract}
The incidence of the placental invasion anomalies are increasing, mainly due to repeat cesarean deliveries. Placenta percreta occurs if these villi perforate the serosa and also sometimes into adjacent organs such as the bladder. The prenatal diagnosis is very important because of the high maternal morbidity and mortality rates without the appropriate surgical planning. The adherent placentas will result in severe early postpartum bleeding, just after the delivery of the fetus. Severe hemorrhage usually results rapidly in disseminated intravascular coagulation (DIC), shock, multiorgan failure or death.The surgery is also challenging due to the risk of the adjacent tissue damage, such as bladder or ureteral injury. Approximately 1 in every 3 cases need intensive care. We present here a prenatally diagnosed, 31-year-old gravida 7 para 2 abortus 4 pregnant case with placenta percreta and rewiev the relevant literature about the key aspects in the ultrasonograhic diagnosis and underlie the key points in the diagnosis.

Keywords: Placental Invasion Anomaly, Placenta Percreta, Obstetric Ultrasound
\end{abstract}

\section{Introduction}

Placental invasion anomalies refer to the abnormal adherence of the placenta to the uterine wall resulting in subsequent detachment failure after the delivery (1). According to the depth of the invasion, there are three sub-groups: placenta accreta occurs if the villi penetrate the decidua but not the myometrium; placenta increta occurs if the villi penetrate through the myometrium; placenta percreta occurs if these villi perforate the serosa and also sometimes into adjacent organs such as the bladder (2). The prevalence is approximately 1 in 2500 pregnancies. However, placenta previa rises the risk more than 10 fold (3). The most frequent predisposing factors are prior cesarean operations and placenta previa. The provenance is rising due to the increase in cesarean operations worldwide (4).

The adherent placentas will result in severe early postpartum bleeding, just after the delivery of the fetus. Severe hemorrhage usually results rapidly in disseminated intravascular coagulation (DIC), shock, multiorgan failure or death. The surgery is also challenging due to the risk of adjacent tissue damage such as ureteral injury (2\%) and bladder injury (15\%). Approximately 1 in every 3 case need intensive care (5). The associated maternal morbidity and mortality risk emphasizes the importance of the prenatal diagnosis. We present here a prenatally diagnosed case of placenta percreta with the emphasis on the importance of the prenatal diagnosis and underlie the key points in the diagnosis.

\section{Case Presentation}

A 31-year-old gravida 7 para 2 abortus 4 pregnant woman was referred at her 32 gestational weeks to our perinatology outpatient clinic due to two prior cesarean deliveries. She never had vaginal bleeding during her pregnancy. Abdominal ultrasound revealed a 32-week fetus with positive cardiac activity. Amniotic fluid was normal. The placenta was lying through the anterior uterine wall with partial closure of the internal cervical os (Figure 1). Due to prior cesarean deliveries and placenta previa partialis with anterior localisation, a targeted ultrasound examination was made and revealed that the retroplacental myometrial zone was non-visible; lacunae could be seen in the lower uterine segment (Figure-1); rich venous blood flow in these intraplacental lacunae was established via color Doppler, the zone between bladder and retroplacental area was irregular (Figure-2). Placenta percreta was our prediagnosis. We scheduled her cesarean operation at 36th gestational week. A pfannenstiel incision was performed. Intraoperative observation of the uterus revealed placenta percreta; but there was no bladder involvement (Figure 3). A longitudinal fundal incision dextrally was made to avoid the placenta (as the placenta was lying through the anterior wall toward the left side), and a 2200 gr female healthy baby was delivered. The umbilical cord was clamped and the placenta was left in the uterus. Hysterectomy was performed without extracting the placenta. Five units of erythrocyte suspensions and 4 units of fresh frozen plasma were given preoperatively. The patient was followed-up in intensive care unit for the first day postoperatively. The postoperative period was uneventful. The patient was discharged at the 7 th day postoperatively. The pathological examination revealed placenta percreta.

\section{Discussion}

The histological changes within the myometrial spiral arterioles which take place as a result of the natural process

Received 7 March 2014, Revised 21 March 2014, Accepted 2 April 2014, Available online 20 July 2014

${ }^{1}$ Assistant Professor, Celal Bayar University School of Medicine, Obstetrics and Gynecology Department, Manisa, Turkey. ${ }^{2}$ Specialist MD, Celal Bayar University School of Medicine, Obstetrics and Gynecology Department, Manisa, Turkey. ${ }^{3}$ Professor, Celal Bayar University School of Medicine, Obstetrics and Gynecology Department, Manisa, Turkey.

*Corresponding Author: Burcu Artunc Ulkumen, Assistant Professor; Celal Bayar University School of Medicine, Obstetrics and Gynecology Department, Manisa, Turkey. Tel: +905324144162,Email: artunc.burcu@gmail.com 


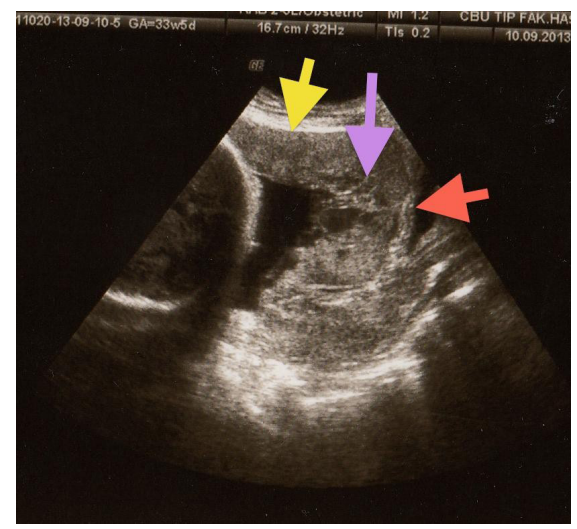

Figure 1. The gray-scale sonographic image of the adherent placenta: the intraplacental lacunae (purple arrow), the irregular border between the placenta and bladder (red arrow), loss of retroplacental clear space (yellow arrow).

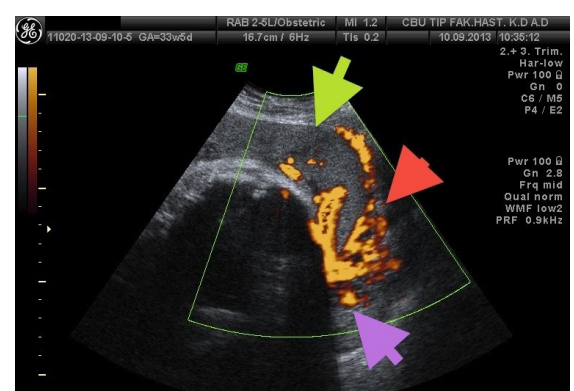

Figure 2. The main diagnostic clues on the color Doppler image of the adherent placenta: loss of retroplacental clear space (yellow arrow), gap in the retroplacental blood flow (red arrow), rich blood flow in placental lacunae (purple arrow).

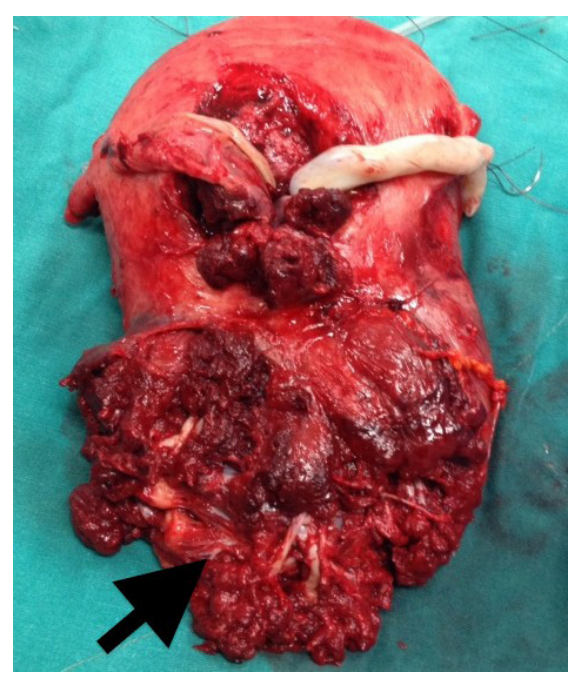

Figure 3. Placenta percreta: postoperative view.

of the pregnancy are different in adherent placentas. The endometrium during pregnancy is defined as the decidua. Decidua basalis (also called decidua placentalis) is the part where the trophoblasts meet the decidual lining. Placental invasion anomalies take place if there is improper development of the Nitabuch's layer with the absence of the decidua basalis. The histological changes in vascular structures like spiral arterioles develop in the deeper layer of the myometrium (2).

The etiology is not well-known. However; multiparity, prior cesarean deliveries, prior abortus history, maternal smoking, prior myomectomy and uterine anomalies are associated with increased risk (6). Prior curettage interventions may affect the endometrial local environment by scar tissue formation and sclerotic changes leading to subsequent defective placentation $(6,7)$. In our case, prior 4 abortion histories and 2 cesarean deliveries were risk factors. Besides, our case was a smoker. Smoking status has been defined as a high risk factor. In a metaanalysis, Faiz et al. proposed that one in four cases would be preventable by quitting smoking (8).

Prenatal diagnosis is important in order to plan the surgical intervention timely. The surgeon has to get the blood products ready, to arrange the anesthesia team for the proper surgery preparation, to inform the patient correctly about the surgical risks, adjacent tissue damage and the need of hysterectomy. Cesarean is planned during 36th gestational week in order to avoid spontaneous severe bleeding and spontaneous labor. Uterine incision is performed vertically in order to avoid the placenta preventing excessive blood loss. If hysterectomy is needed, placenta should be left in the uterus and the next surgical steps should be initiated.

Ultrasonography is the main tool in diagnosis. It detects up to $80 \%$ of the cases (9). During the $18-23$ week detailed sonographic examination, the risk factors should be evaluated for each pregnant woman. Regarding highrisk pregnancies with prior cesarean operations, prior curettage history or current placenta previa; a targeted sonographic examination would be reasonable to detect placental invasion anomalies (10).

In 1980's, Tabsh et al. and Mondenca et al. were the first describing some typical sonographic profiles of placenta accreta $(11,12)$. They identified that the natural peripheric hypoechoic zone between placenta and myometrium could not be visualized in adherent placentas. HoffmanTretin and co-workers described some additional features: multiple intra-placental lakes and progressive thinning of the retroplacental hypoechoic myometrial zone on each subsequent visit (13). Finberg et al. identified two additional sonographic profiles for placenta percreta cases: a thinning or focal disruption area of the uterine serosa-bladder wall complex and the appearance of a focal mass beyond the uterine serosa, with the same echogenicity of placenta (14). Lerner and co-workers (15) were the first who tried to diagnose the adherent placenta antenatally by color doppler and transvaginal sonography. Chou et al. showed dense and highly pulsatile blood flow within placental lacunae (16). MRI can be helpful in posteriorly located adherent placentas and in unclear sonographic profiles (10).

In our perinatology outpatient clinic, we perform a targeted ultrasound examination for low uterine segment for high-risk pregnancies during the detailed screening between 18-23 gestational weeks. We evaluate firstly the placental localisation. We remark the intraplacental 
anechoic areas: lacunae are vascular structures with different size and shapes especially prominent in the third trimester having turbulent flow within. Placental lakes are round shaped anechoic areas with laminar blood flow in it and with uneventful outcomes (10). We carefully evaluate the normal clear zone between placenta and myometrium. Loss of this zone may indicate the adherent placenta. After that, we evaluate the suspected zone also with color doppler: any gap in the myometrial blood flow in the retroplacental area may be alarming about the adherent placenta. In order to evaluate the region between the bladder and uterine serosa, the bladder needs to be full. We prefer transabdominal sonographic examination during the daily practice. However, Lerner et al suggested that transvaginal sonography may enhance the accurate antenatal diagnosis of the adherent placentas. Transvaginal sonography has better near-field resolution power for the evaluation of the region between the placenta and lower uterine segment (15). We prefer transvaginal sonography if we could not evaluate the lower uterine segment properly (especially in obese women with prior cesarean) or if the placenta has posterior location.

Recent studies showed that visualization of placental lacunae had the highest sensitivity (93\%) for the detection of placenta accreta between 15-40 gestational weeks. In contrast to placental lakes with rounded and smooth bordered shapes, lacunae tend to be more linear with irregular borders. Placenta with lacunae has typical 'moth-eaten' appearance (17). Similarly, we found on 2-dimensional gray-scale sonographic examination irregular placental lacunae with rough borders. On color doppler, there was turbulent flow in these lacunae. We also found bulging and interruption of the integrity between the bladder and uterine wall which may also warned us about the adherent placenta; however, also a prior cesarean operation with the retraction of the bladder peritoneum before the uterine incision could have resulted in the increase of the vascularity in that area which may have caused an impression of placenta percreta.

\section{Conclusion}

As a result, the turbulent blood flow in placental lacunae especially in the third trimester, loss of retroplacental anechoic regular zone, rich and irregular blood flow into the adjacent tissues from the placenta should warn the clinician about the risk of the adherent placenta. In case of unclear diagnosis, MRI may be helpful.

\section{Ethical issues}

The local ethics committee approved the study.

\section{Conflict of interests}

None.

\section{References}

1. Kent A. Management of placenta accreta. Rev Obstet Gynecol 2009; 2(2): 127- 8.

2. Callen PW. Ultrasound evaluation of the placenta and umbilical cord. In: Feldstein VA, et al (ed). Ultrasonography in obstetrics and gynecology. 5th edition Saunders,Elsevier;2008. p. 737-39.

3. Morgan K, Arulkumaran S. Antepartum haemorrhage. Curr Obstet Gynecol 2003; 13: 81-87.

4. Yeniel AÖ, Ergenoglu M, Zeybek B, Karadadas N. Placenta previa percreta. Ege Journal of Medicine 2010; 49(2):123-127.

5. Silver RM, Landon MB, Rouse DJ, Leveno KJ, Spong CY, Thom EA, et al. Maternal morbidity associated with multiple repeat cesarean deliveries. Obstet Gynecol 2006; 107:1226-1232.

6. Tuzovic L, Djelmis J, Ilijic M. Obstetric Risk Factors Associated with Placenta Previa Development: CaseControl Study. CMJ 2003; 44(6):728-733.

7. Sheiner E, Shoham-Vardi I, Hallak M, Hershkowitz R, Katz M, Mazor M. Placenta previa: obstetric risk factors and pregnancy outcome. J Matern Fetal Med 2001; 10:414-9.

8. Faiz AS, Ananth CV. Etiology and risk factors for placenta previa: an overview and meta- analysis of observational studies. J Matern Fetal Neonatal Med 2003; 13:175- 190.

9. Warshak CR, Eskander R, Hull AD, Scioscia AL, Mattrey RF, Benirschke K, et al. Accuracy of ultrasonography and magnetic resonance imaging in the diagnosis of placenta accreta. Obstet Gynecol 2006; 108:573-581.

10. Baughman WC, Corteville JE, Shah RR. Placenta Accreta: Spectrum of US and MR Imaging Findings. Radio Graphics 2008; 28(7):1905-16.

11. Tabsh KMA, Brinkman CR, King W. Ultrasound diagnosis of placenta increata. J Clin Ultrasound 1982; 10:288.

12. Mendonca LK. Sonographic diagnosis of placenta accreata, presentation of six cases. J Ultrasound Med 1988; 7: 211-15.

13. Hoffman-Tretin JC, Koenigsberg M, Rabin A, Anyaegbunam A. Placenta accreta: additional sonographic observations. J Ultrasound Med 1992; 11:29-34.

14. Finberg HJ, Williams JW. Placenta accreata: prospective sonographic diagnosis in patients with placenta previa and prior cesarean section. J Ultrasoun Med 1992; 11:333-43.

15. Lerner JP, Deane S, Timor-Tritsch IE. Characterization of placenta accreata using transvaginal sonography and color doppler imaging. Ultrasound Obstet Gynecol 1995; 5:198-201.

16. Chou MM, Ho ES, Lee YH. Prenatal diagnosis of placenta previa/accreta with color doppler ultrasound. Ultrasound Obstet Gynecol. 2000;15(1):28-35.

17. Comstock $\mathrm{CH}$. Antenatal diagnosis of placenta accreta: a review. Ultrasound Obstet Gynecol 2005; 26:89-96.

Copyright ( 2014 The Author(s); This is an open-access article distributed under the terms of the Creative Commons Attribution License (http://creativecommons.org/licenses/by/4.0), which permits unrestricted use, distribution, and reproduction in any medium, provided the original work is properly cited. 